\title{
Comparison of the Null Distributions of Weighted Kappa and the C Ordinal Statistic
}

\author{
Domenic V. Cicchetti \\ West Haven VA Hospital and Yale University \\ Joseph L. Fleiss \\ Columbia University
}

\begin{abstract}
It frequently occurs in psychological research that an investigator is interested in assessing the extent of interrater agreement when the data are measured on an ordinal scale. This monte carlo study demonstrates that the appropriate statistic to apply is weighted kappa with its revised standard error. The study also demonstrates that the minimal number of cases required for the valid application of weighted kappa varies between 20 and 100 . depending upon the size of the ordinal scale. This contrasts with a previously cited large sample estimate of 200 . Given the difficulty of obtaining sample sizes this large, the latter finding should be of some comfort to investigators who use weighted kappa to measure interrater consensus.
\end{abstract}

\section{Introduction}

Suppose that two raters independently use the same $k$-point ordinal scale to evaluate $N$ subjects. The results of these $N$ pairs of evaluations may be summarized in a $k \times k$ contingency table in which the cell entries are the proportions of subjects given each of the $k^{2}$ possible dual ratings. The statistic weighted kappa was proposed by Cohen (1968) as a measure of agreement between the two raters when the relative seriousness of each possible disagreement could be quantified.

APPLIED PSYCHOLOGICAL MEASUREMENT

Vol. 1. No. 2 Spring 1977 pp. 195-201

() Copyright 1977 West Publishing Co.
Let $\mathrm{P}_{i j}$ be the proportion of subjects assigned to category $i$ by rater 1 and to category $j$ by rater 2 ; let

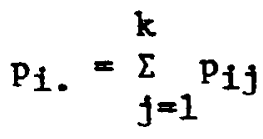

be the proportion of subjects assigned to the $i$ th category by rater 1 ; and let

$p_{. j}=\sum_{i=1}^{k} p_{i j}$

be the proportion of subjects assigned to the $j$ th category by rater 2 . Finally, let $w_{i j}$ be the agreement weight given the $i, j$ th cell where

$0 \leqslant \mathrm{w}_{i j} \leqslant 1$ and $\mathrm{w}_{i i}=1$ for all $i=1, \ldots, k$.

The observed weighted proportion of rater agreement is given by

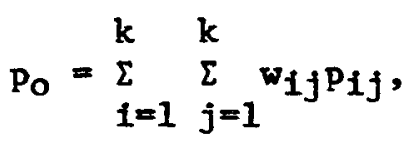

and the chance-expected weighted proportion of agreement by

$$
P_{C}=\sum_{i=1}^{k} \sum_{j=1}^{k} w_{i j} P_{i} . P_{. j} \cdot
$$


Then, weighted kappa is defined by

$$
\hat{k}_{w}=\frac{p_{o}-P_{c}}{1-p_{c}} \text {. }
$$

Weighted kappa varies from negative values for poorer than chance agreement through zero for just chance agreement to +1 for perfect agreement.

The $C$ statistic for ordinal scale agreement (Cicchetti, 1972a; 1972b) is a special case of the numerator of weighted kappa when the weights are given by

$$
w_{i j}=1-\frac{|i-j|}{k-1}
$$

thus,

$\mathrm{C}=$

$$
\frac{\sum_{i=1}^{k} \sum_{j=1}^{k}|i-j|\left(P_{i} \cdot P_{. j}-P_{i j}\right)}{k-1}
$$

Fleiss, Cohen \& Everitt (1969) found the large sample standard error of weighted kappa to be estimable by

$$
\begin{aligned}
\text { S.E. }\left(\hat{\mathrm{k}}_{\mathrm{w}}\right) & =\frac{1}{\left(1-p_{c}\right) \sqrt{\mathrm{N}}} \\
& \sum_{i=1}^{k} \sum_{j=1}^{k} p_{i \cdot p_{. j}\left(w_{i j}-\left(\bar{w}_{i}+\bar{w}_{. j}\right)\right)^{2}-p_{c}{ }^{2},}^{1 / 2}
\end{aligned}
$$

where

$$
\bar{w}_{i}=\sum_{j=1}^{k} w_{i j} p_{\cdot j}
$$

is a weighted average of the weights in the $i$ th row and

$$
\bar{w}_{\cdot j}=\sum_{i=1}^{k} w_{i j} p_{i}
$$

is a weighted average of weights in the $j$ th column. The hypothesis that agreement is significantly better than chance may be tested by re- ferring the critical ratio

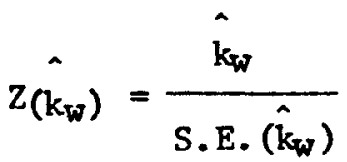

to the standard normal distribution.

Cicchetti (1972a) proposed

$$
\begin{aligned}
& \text { k } k \\
& \text { S.E. }(C)=\left[\left(\sum_{i=1} \sum_{j=1} w_{i j}^{2} p_{i j}\right.\right. \\
& \left.\left.-\sum^{k} \sum^{k}\left(w_{i j} p_{i j}\right)^{2}\right) \div(N-1)\right]^{1 / 2} \\
& i=1 \quad j=1
\end{aligned}
$$

as an approximate standard error for the $C$ statistic, and proposed that the resulting critical ratio

$$
Z(C)=\frac{P_{0}-P_{c}}{\text { S.E. (C) }}
$$

be referred to the standard normal distribution in order to test whether agreement was significantly better than chance.

Because the critical ratios given by Equations 11 and 13 are quite different, a monte carlo study was conducted in order to determine which critical ratio was more nearly normally distributed, and how the approach to normality depends on (1) the number of points, $k$, on the scale; (2) the number of subjects; and (3) the pattern of marginals for one observer relative to the other.

\section{Method}

The sampling distributions of the critical ratios in Equations 11 and 13 were studied by simulation under the hypothesis that the assignments by the two raters were independent.

The following parameters were systematically varied:

1. The number of points on the scale, $k$, ranged between 3 and 7 .

2. The number of subjects, $N$, ranged between approximately $k^{2} / 2$ and $16 k^{2}$.

3. Let $\left(\pi_{i}, \pi_{j} ; i, j=1, \ldots, k\right)$ denote the under- 
lying marginal probabilities used to generate a set of tables. For each value of $k$ and $N$, three pairs of marginal probabilities were studied:

(a) uniform marginals $\left(\pi_{i .}=\pi_{. j}=1 / k\right.$ for all $i$ and $j$ );

(b) moderately different marginals with

k

$\sum\left|\pi_{i} .-\pi_{. i}\right| / k$

$i=1$

ranging between .04 and .10 depending on the value of $k$; and

(c) markedly different marginals with values derived from Equation 14 ranging between .14 and .40 . In this condition, the underlying marginal probabilities for rater 1 were taken to be the exact reverse of those for rater 2. For the three-point scale, for example, rater 1's marginal proportions were $.7, .2$, and .1 , while the corresponding proportions for rater 2 were $.1, .2$, and .7.

Under each combination of the parameters identified above, between 1000 and 8000 tables (depending on the value of $N$ ) were generated at random by a program written for the IBM 360. For each table, the critical ratios in Equations 11 and 13 were calculated; the weights used in calculating $\hat{\mathbf{k}}_{w}$ and $C$ were those specified in Equation 6.

The empirical distributions of the two critical ratios were compared with the theoretical normal distribution in terms of the mean, variance, skewness $\left(\beta_{1}\right)$ and kurtosis $\left(\beta_{2}\right)$, for which the theoretical values are respectively $0,1,0$, and 3 , and in terms of selected one- and two-tailed areas.

\section{Results}

Since the general findings of this monte carlo study were the same for all five values of $k$ investigated $(3 \leqslant k \leqslant 7)$, results will be presented for the 5-point ordinal scale only.
1. Under conditions of uniform marginal proportions, the $Z$ distributions for both $C$ and $\overrightarrow{\mathbf{k}}_{w}$ produce the best results. However, the observed moments for $\mathbf{k}_{w}$ more closely approximate the expected values of mean $=0$, variance $=1, \beta_{1}=0$, and $\beta_{2}=3$. These results are best for $N \geqslant 2 k^{2}$.

2. When the differences in the rater marginals are moderate, the $Z$ of $\overrightarrow{\mathrm{k}}_{i}$ continues to produce acceptable results with the variances much closer to the theoretical value of 1 than when S.E. $(C)$ is applied. These results also tend to hold for $N \geqslant 2 k^{2}$.

3. For extreme differences in the rater marginals, the standard error for $\overline{\mathbf{k}}_{\mathrm{w}}$ even more clearly produces the better results. The major difference between the two distributions is that $Z$ based upon the Fleiss, et al. standard error consistently produces variance values not departing appreciably from 1 , while the $Z$ based upon the $C$ standard error produces very low variances, approximately in the range of .3 to .4. Thus, even under conditions of markedly different interrater marginal proportions, the Fleiss, et al. standard error holds up admirably well. Once again, the two approaches work best with $N \geqslant 2 k^{2}$. These results are illustrated in Table 1.

The results of these analyses indicate that the critical ratio $Z_{\left(\hat{k}_{w}\right)}$ is always superior to $Z(C)$ and thus that S.E. $(C)$ is invalid. When the observed probabilities of the $Z_{\left(\bar{k}_{w}\right)}$ and $Z(C)$ distributions were compared to the one- and two-tailed area probabilities previously cited, the results, as given in Table 2, tended to closely parallel those based upon the first four moments. Once again, these results indicate that S.E. $\left(\hat{k}_{w}\right)$ is superior to S.E. $(C)$ and thus is the valid formula for assessing levels of statistical significance for $\overline{\mathrm{k}}_{w}$.

\section{Discussion and Conclusions}

The results of this study are quite easy to interpret. It is clear that when assessing rater agreement with ordinal data, the weighting sys- 
Table 1

Central Moments of Nu1l Distributions

of Two Critical Ratios for a $5 \times 5$ Table

A. Uniform Rater Marginals ${ }^{a}$

\begin{tabular}{|c|c|c|c|c|c|c|c|c|c|c|c|}
\hline \multirow{2}{*}{$\begin{array}{l}\text { Central } \\
\text { Moments } \\
\text { Mean }\end{array}$} & \multirow{2}{*}{$\begin{array}{c}\begin{array}{c}\text { Expected } \\
\text { Values }\end{array} \\
0\end{array}$} & \multirow{2}{*}{$\begin{array}{r}\mathrm{N}=24 \\
.04\end{array}$} & \multirow{2}{*}{$\begin{array}{r}\mathrm{N}=50 \\
.02\end{array}$} & \multicolumn{2}{|c|}{$\begin{array}{l}\text { C Statistic: } \\
N=100 \quad N=200\end{array}$} & \multirow{2}{*}{$\begin{array}{r}N=400 \\
.03\end{array}$} & \multirow{2}{*}{$\begin{array}{c}\mathrm{N}=24 \\
.02\end{array}$} & \multirow{2}{*}{$\begin{array}{r}N=50 \\
.01\end{array}$} & \multicolumn{3}{|c|}{$\begin{array}{l}\text { We1ghted kappa: } \\
N=100 \quad N=200 \quad N=400\end{array}$} \\
\hline & & & & .02 & .02 & & & & .02 & .02 & .03 \\
\hline Varlance & 1 & .91 & .87 & .85 & .85 & .84 & 1.07 & 1.03 & 1.00 & 1.00 & .99 \\
\hline$\beta_{1}$ & 0 & .19 & .10 & .05 & .04 & .05 & .08 & .04 & .01 & .01 & .04 \\
\hline$B_{2}$ & 3 & 3.32 & 3.19 & 3.15 & 3.23 & 2.94 & 2.82 & 2.95 & 2.98 & 3.14 & 2.91 \\
\hline
\end{tabular}

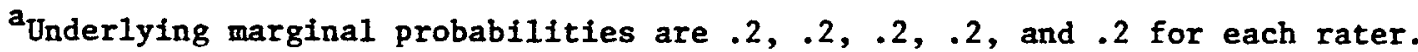

B. Moderate Differences in Rater Marginals ${ }^{b}$

\begin{tabular}{lrrrrrrrrrrr}
\hline \hline Mean & 0 & .04 & .03 & .03 & .04 & .05 & .01 & .02 & .03 & .04 & .05 \\
Variance & 1 & .76 & .73 & .72 & .73 & .76 & 1.06 & 1.05 & 1.03 & 1.05 & 1.09 \\
$\beta_{1}$ & 0 & .27 & .17 & .11 & .08 & .11 & .18 & .13 & .09 & .06 & .12 \\
$\beta_{2}$ & 3 & 3.91 & 3.22 & 3.09 & 3.03 & 3.04 & 2.92 & 2.89 & 2.91 & 2.95 & 2.97 \\
\hline
\end{tabular}

bonderlying marginal probabllitles are $.35, .20, .20, .15$, and .10 for Rater 1 , and $.40, .30, .10, .10$, and .10 for Rater 2 .

c. Marked Differences in Rater Marginals ${ }^{c}$

\begin{tabular}{lrrrrrrrrrrr}
\hline \hline Mean & 0 & .01 & .00 & .00 & .01 & .02 & .00 & .02 & .02 & .03 & .03 \\
Variance & 1 & .33 & .33 & .32 & .33 & .33 & 1.05 & 1.04 & 1.03 & 1.04 & 1.05 \\
$B_{1}$ & 0 & -.05 & -.04 & -.02 & -.01 & .01 & -.17 & -.15 & -.06 & .00 & .10 \\
$B_{2}$ & 3 & 3.97 & 3.32 & 3.05 & 3.04 & 2.82 & 2.73 & 2.82 & 2.84 & 2.92 & 2.75 \\
\hline
\end{tabular}

CUnderlying marginal probabilities are $.45, .20, .20, .10$, and .05 for Rater 1 , and $.05, .10, .20, .20$, and .45 for Rater 2 . 
Empirical Tail Areas of Null Distributions

of Two Critical Ratios for a $5 \times 5$ Table

A. Uniform Rater Marginals

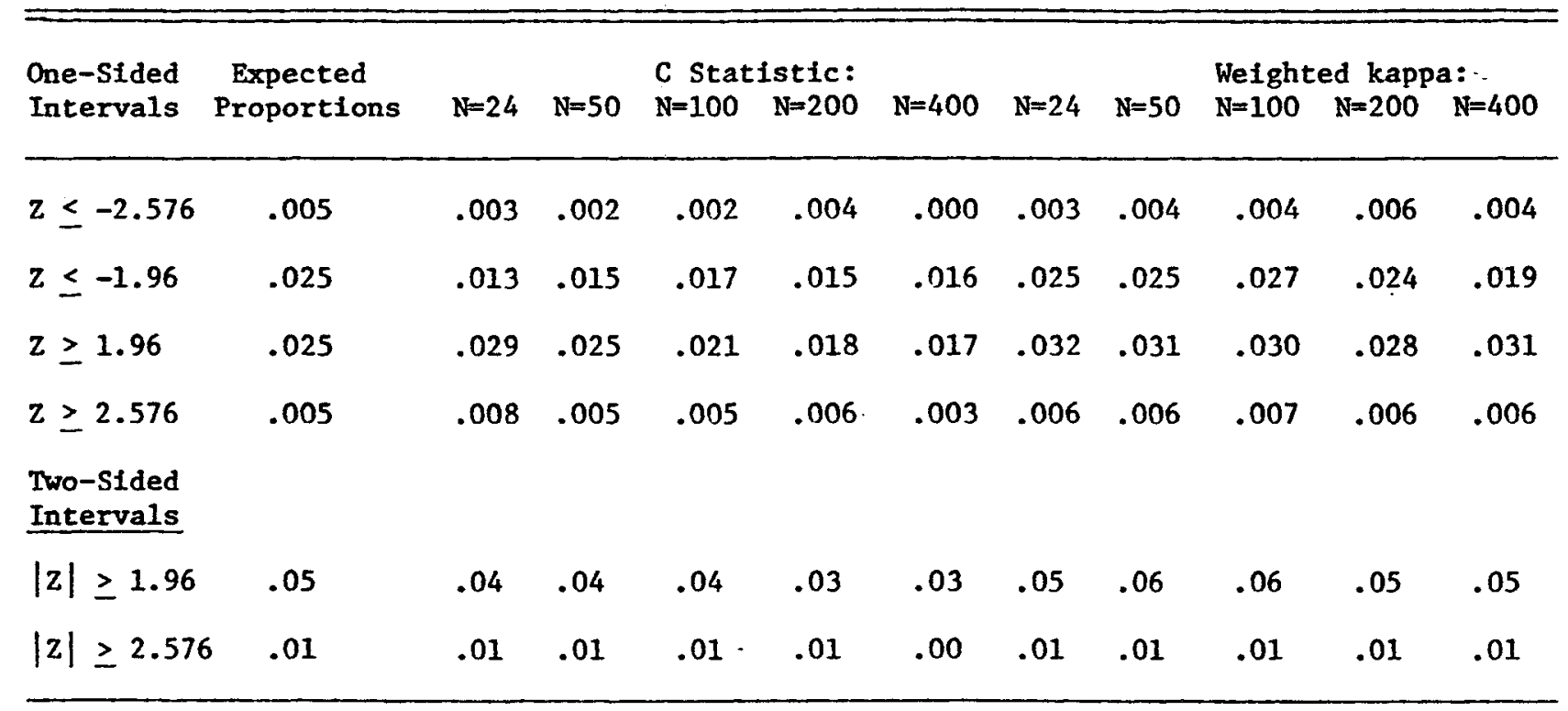

anderlying marginal probabilities are $.2, .2, .2, .2$, and .2 for both Raters.

B. Moderate Differences in Rater Marginals ${ }^{b}$

\begin{tabular}{|c|c|c|c|c|c|c|c|c|c|c|c|}
\hline$z \leq-2.576$ & .005 & .001 & .000 & .000 & .000 & .000 & .002 & .004 & .004 & .005 & .005 \\
\hline$z \leq-1.96$ & .025 & .007 & .006 & .007 & .007 & .009 & .022 & .021 & .020 & .025 & .024 \\
\hline$z \geq 1.96$ & .025 & .023 & .019 & .015 & .015 & .018 & .033 & .033 & .029 & .032 & .034 \\
\hline$z \geq 2.576$ & .005 & .008 & .004 & .003 & .003 & .005 & .008 & .009 & .007 & .008 & .011 \\
\hline \multicolumn{12}{|l|}{$\begin{array}{l}\text { Two-Sided } \\
\text { Intervals }\end{array}$} \\
\hline$|z| \geq 1.96$ & .05 & .03 & .02 & .02 & .02 & .03 & .05 & .05 & .05 & .06 & .06 \\
\hline$|z| \geq 2.576$ & .01 & .01 & .00 & .00 & .00 & .01 & .01 & .01 & .01 & .01 & .02 \\
\hline
\end{tabular}

bunderlying marginal probabilities are $.35, .20, .20, .15$, and .10 for Rater 1 , and $.40, .30, .10, .10$, and .10 for Rater 2 . 
C. Marked Differences in Rater Marginals ${ }^{c}$

\begin{tabular}{|c|c|c|c|c|c|c|c|c|c|c|c|}
\hline$z \leq-2.576$ & .005 & .001 & .000 & .000 & .000 & .000 & .007 & .006 & .007 & .007 & .000 \\
\hline$z \leq-1.96$ & .025 & .003 & .002 & .001 & .001 & .000 & .032 & .029 & .025 & .026 & .025 \\
\hline$z \geq 1.96$ & .025 & .001 & .000 & .000 & .000 & .000 & .018 & .023 & .026 & .030 & .031 \\
\hline$z \geq 2.576$ & .005 & .000 & .000 & .000 & .000 & .000 & .003 & .003 & .004 & .004 & .008 \\
\hline \multicolumn{12}{|l|}{$\begin{array}{l}\text { Two-Sided } \\
\text { Intervals }\end{array}$} \\
\hline$|z| \geq 1.96$ & .05 & .04 & .00 & .00 & .00 & .00 & .05 & .05 & .05 & .06 & .06 \\
\hline$|z| \geq 2.576$ & .01 & .01 & .00 & .00 & .00 & .00 & .01 & .01 & .01 & .01 & .01 \\
\hline
\end{tabular}

Underlying marginal probabilities are $.45, .20, .20, .10$, and .05 for Rater 1 , and $.05, .10, .20, .20$, and .45 for Rater 2 .

tem defined in Equation 6 should be used with the standard error developed for weighted kappa by Fleiss, et al. (1969), and that the critical ratio should be defined by Equation 11 . The results of this monte carlo study indicate that this statistical approach is valid even for $N$ as small as $2 k^{2}$. This is a particularly important finding, since it represents a much smaller range of $N$ for the usual 3-, 4-, 5-, 6-, or 7-point ordinal scales (i.e., approximately 20 for the 3 -point scale to about 100 for the 7 -point scale). This contrasts with the more conservative estimate of $N \geqslant 200$ due to Fleiss, Cohen, \& Everitt (1969). Given the difficulty of obtaining sample sizes of $\geqslant 200$, this finding should be of some comfort to investigators using these statistical approaches.

In summary, a large scale monte carlo study has shown that weighted kappa and its standard error may safely be used to assess rater agreement with ordinal data. The weighting system will vary as a function of the substantive nature of the ordinal scale.

It should be noted that both Fleiss \& Cohen (1973) and Krippendorff (1970) have shown the conditions under which weighted kappa and the intraclass correlation coefficient are mathematically equivalent. The latter statistic is the appropriate approach to the problem of assessing rater reliability when the data are continuous.
Thus, we appear to be at the point in which we have available a "family" of valid, interrelated statistics which can easily be modified to fit the case of assessing observer agreement for different types of research data, both ordinal and continuous.

\section{References}

Cicchetti, D. V. A new measure of agreement between rank ordered variables. Proceedings of the American Psychological Association, 1972, 7, 17-18. (a)

Cicchetti, D. V. Assessing observer agreement with dichotomous-ordinalized data. Biometrics. 1972, 28. 1165 (Abstract). (b)

Cohen, J. Weighted Kappa: nominal scale agreement with provision for scaled disagreement or partial credit. Psychological Bulletin. 1968, 70, 213-220.

Fleiss, J. L., \& Cohen, J. The equivalence of weighted kappa and the intraclass correlation coefficient as measures of reliability. Educational and Psychological Measurement, 1973, 33, 613-619.

Fleiss, J. L., Cohen, J., \& Everitt, B. S. Large sample standard errors of kappa and weighted kappa. Psychological Bulletin. 1969, 72, 323-327.

Krippendorff, K. Bivariate agreement coefficients for reliability of data. In E. G. Borgatta (Ed.), Sociological Methodology. San Francisco: Jossey-Bass, 1970.

\section{Acknowledgements}

This research w'as supported by the West Haven $V A$ Hospital (MRIS 1416). The preliminary findings 
COMPARISON OF THE NULL DISTRIBUTIONS OF

were presented at an invited paper session, chaired by Dr. Fleiss at the Joint Central Meetings of the American Statistical Association. St. Paul. Minnesota, March. 1975. The authors wish to acknowledge the major role played by Joseph Vitale, at Yale University, in developing the computer programs used in this research. Appreciation is also extended to Dr. Barry Margolin. Yale University. for his expert counsel, critical review, and encouragement, during the early phases of this research.

\section{Authors' Addresses}

Dominic V. Cicchetti, Research Psychologist, VA Hospital, West Haven, Connecticut 06516. Joseph L. Fleiss, Professor and Head, Division of Biostatistics, Columbia University. School of Public Health. 600 West 168th Street. New York, New York 10032 